\title{
Personalized Search Engine for Mobiles Using Location Accuracy and Privacy
}

\author{
Nidhi Parikh \\ Kit" s College Of Engineering, Shivaji University, Gokul Shirgaon, Kolhapur, Maharashtra 416012
}

\begin{abstract}
The proposed system of a Personalized Search Engine For Mobile Using Location Accuracy And Privacy takes user's preferences and data in the form of concepts by analyzing their click through data. User's locations (positioned by GPS) are used to give the location concepts. Depending upon threshold value and users interest the score is calculated for each of the urls that user has visited. Depending upon the score the preferred page is displayed to the user.
\end{abstract}

Keywords: personalization, locations, search engine, optimization.

\section{Introduction}

Todayes technology has gifted us a very efficient way of making the things very easier for human beings. Android is a very common platform used by majority of mobile users, it is a mobile operating system based on the Linux kernel with a user interface designed primarily for smart phones. A smart phone uses number of applications including various search engines like Google, Yahoo, Bing and so on, however they have certain limitations. As we know that major problem in mobile search is that the interactions between the users and search engines are limited by the small factors of the mobile devices. This problem includes submitting short queries and comparing it with web search engine which results in rise of ambiguity. Main concern is to return relevant results to the users according users preferences. We propose a design for android phones that will take into consideration users profile along with its interests and assess the search results according to the users"e profiles. This mobile search engine will exclusively analyze user interest and obtain prompt results to user. Assuming the need for new concepts i introduce here concept called "Personalization". It will take into account the physical locations of user in our search engine using GPS technology. The GPS locations helps reinforcing the user"s location preferences derived from a user's search activities to provide the most relevant results .The results show that GPS locations helps in effective retrieval for location queries.

\section{Existing Techniques}

- Existing location-based search systems require users to manually define their location preferences.

- We propose and implement a new and realistic design that will train the user profiles quickly and efficiently to handle the training and re ranking processes.

- Existing works on personalization do not address the issues of privacy preservation.

\section{Contribution}

\subsection{Creation of Android Application}

The implementation of first module is developed by androidadt bundle tool kit. The GUI for Login, Registration and
Submission of the query is designed. The new user of the application has to first register and then login so as to submit the query to the search engine. After successful login of the user, if the user is searching the query for the first time, it has to go to the training activity in the application. In the training activity the user will submit a query of his choice for his search and select a url from the list obtained by server. The testing activity displays the click through of the user. The probability activity displays the most probable results for the query.

3.2 Maintaining Log Record according to the users' preference on the server

The main purpose of Android Application is to provide „Registration ${ }^{\text {ee }}$ and „Logine to the user of the application. This is achieved by designing ,Registration "e and „Login "e activities using java programming language. The input given by the User stored in the ,Relational Database ${ }^{e c}$ with table name „Registration ${ }^{\text {ee }}$ using „Oracle ${ }^{e e}$ Database. The android interface takes the query from the user and sends the query to the backend search engine and stores the results in the database. This module stores and maintains the data in the database. The results obtained from the backend search engine are stored on the drive as an html file. We have created different database tables for maintaining different records. The snippet obtained from the backend search engine is stored in the drive on the server machine. This is done by writing a JSP Code.

3.3 The Re ranking the search results at Optimized Personal Search Engine server

The Re ranking module carries out the function of re-ranking of the results obtained from the backend search engine and then performs analyzing and processing the previously stored results in the database. The re ranking of the data is done according to the steps of the algorithm defined below. This process of re ranking involves scanning of the snippet to extract the keywords and calculating weights for every keyword, calculating support to find the preferable and most probable results to the user. This module is implemented in a java files and JSP pages.

\section{Volume 4 Issue 12, December 2015}




\section{International Journal of Science and Research (IJSR) \\ ISSN (Online): 2319-7064}

Index Copernicus Value (2013): 6.14 | Impact Factor (2014): 5.611

\subsection{Display the Re-ranked Results to the user}

Finally the re-ranked results are displayed to the user by using the spinner in Android Development Platform which shows the list of the URLs to the user along with the concept of personalization, that is only those URLs in the database are shown to the user which he has earlier searched for otherwise the message of match being not found is displayed. The JSP is used for finding probability and fetching URL whose count is highest.

\section{Results}

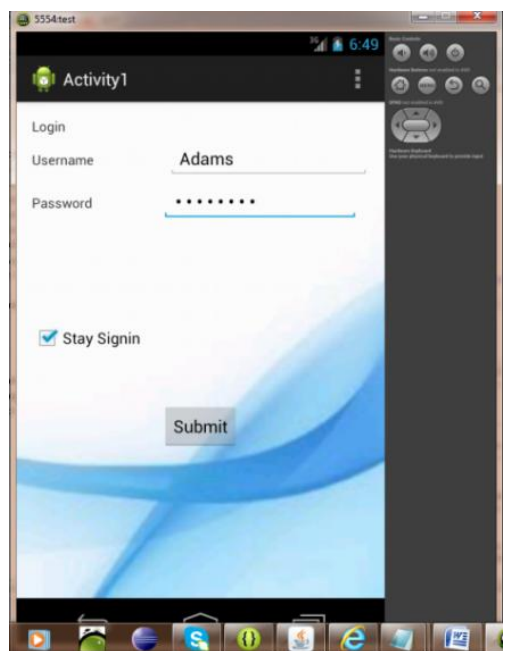

Figure 1: The user has to login by his/her Username and password.
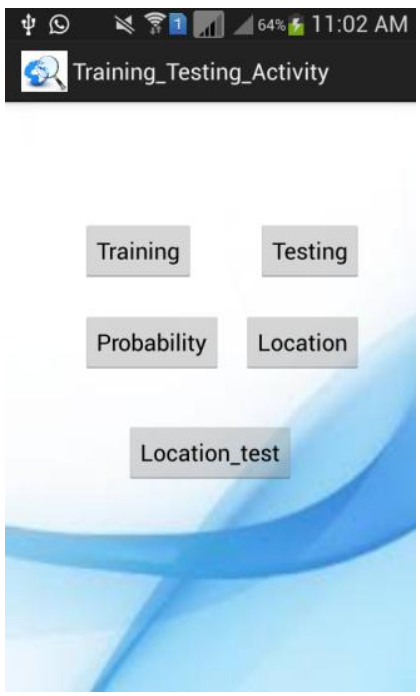

Figure 2: The training testing activity provides options for searching based on the content features.

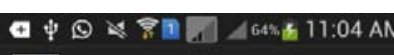

Q. Activity 2

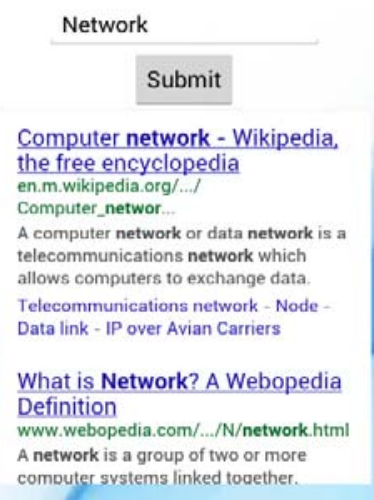

Figure 3: This activity collects the users click through data for re-ranking purpose
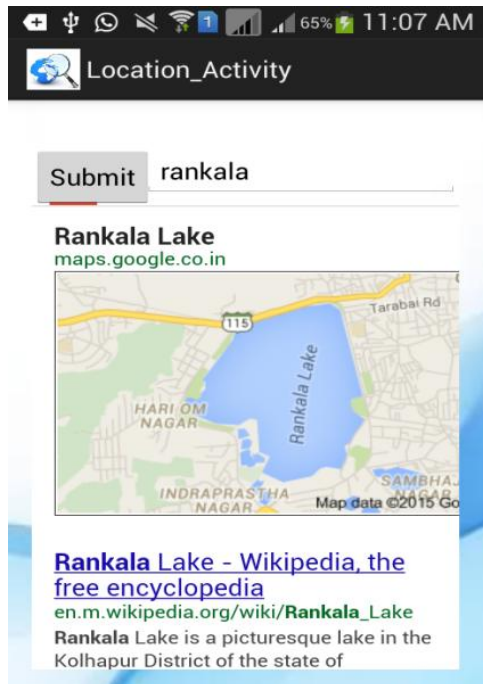

Figure 4: This activity performs search considering the current location of the user.

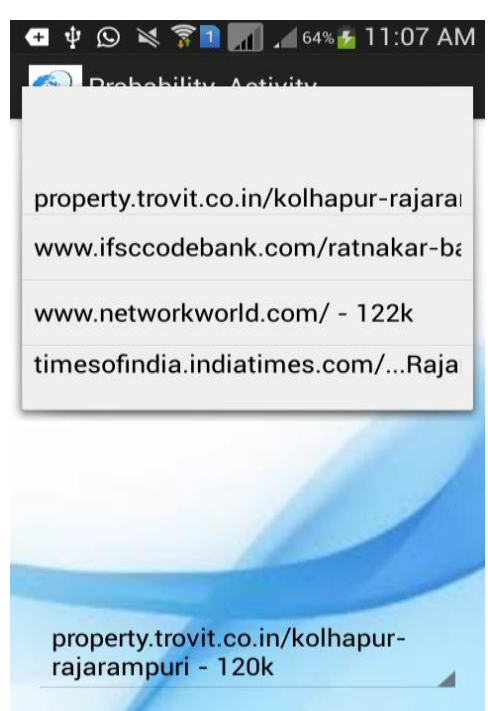

Figure 6: This activity shows the results based on the current location of the users. These are the results obtained after tracing the current location of the users 


\section{Conclusion}

This project "Personalized Search Engine For Mobiles Using Location Accuracy And Privacy" is an implementation of personal search in optimized form. The system overcomes the drawbacks of the current system by providing privacy through username and provides faster and optimized way of search. The application makes the use of the GPS facility in the android phones for tracing the location of the user. We observed that results are correct up to $95 \%$ for the test which we carried out.

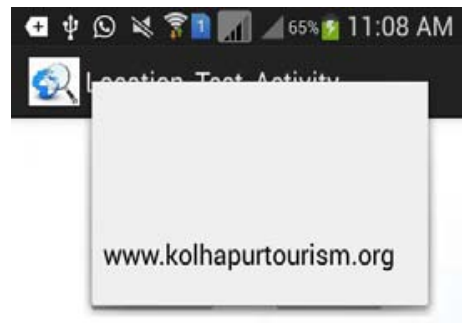

Figure 5: This activity shows the most probable results for a search performed by the user. These are the final results after analyzing the content features

\section{References}

[1] K.W.-T. Leung, D.L. Lee, and W.-C. Lee, "Personalized Web Search with Location Preferences," Proc. IEEE Int' 1 Conf. Data Mining (ICDE), 2010.

[2] H. Li, Z. Li, W.-C. Lee, and D.L. Lee, "A Probabilistic Topic-Based Ranking Framework for Location-Sensitive Domain Information Retrieval," Proc. Int'1 ACM SIGIR Conf. Research and Development in Information Retrieval (SIGIR), 2009

[3] Q. Gan, J. Attenberg, A. Markowetz, and T. Suel, "Analysis of Geographic Queries in a Search Engine Log," Proc. First Int" 1 Workshop Location and the Web (LocWeb), 2008.

[4] Appendix, http:/www.cse.ust.hk/faculty/dlee/tkdepmse/ appendix.pdf, 2012 Nat'1 geospatial, http://earthinfo.nga.mil/, 2012. 